\title{
Enhanced Performance of Li-ion Batteries via Modified Reference Governors \& Electrochemical Models
}

\author{
H. E. Perez, Student Member, IEEE, N. Shahmohammadhamedani, and S. J. Moura, Member, IEEE
}

\begin{abstract}
This paper examines reference governor (RG) methods for satisfying state constraints in Li-ion batteries. Mathematically, these constraints are formulated from a first principles electrochemical model. Consequently, the constraints explicitly model specific degradation mechanisms, such as lithium plating, lithium depletion, and overheating. This contrasts with the present paradigm of limiting measured voltage, current, and/or temperature. The critical challenges, however, are that (i) the electrochemical states evolve according to a system of nonlinear partial differential equations, and (ii) the states are not physically measurable. Assuming available state and parameter estimates, this paper develops RGs for electrochemical battery models. The results demonstrate how electrochemical model state information can be utilized to ensure safe operation, while simultaneously enhancing energy capacity, power, and charge speeds in Li-ion batteries.
\end{abstract}

Index Terms-Batteries, Electrochemical Modeling, Reference Governor, Constrained Control, Performance Benefits Comparison.

\section{INTRODUCTION}

$\mathbf{T}$ HIS paper develops a reference governor-based approach to operating lithium-ion batteries at their safe operating limits.

Battery energy storage is a key enabling technology for portable electronics, electrified transportation, renewable energy integration, and smart grids. A crucial obstacle to the proliferation of battery energy storage is cost. Specifically, battery packs are typically oversized and underutilized to ensure longevity and robust operation. Indeed, oversizing mitigates several degradation mechanisms, such as lithiumplating, lithium depletion/over-saturation, overheating, and stress fractures by reducing C-rates ${ }^{1}$. However, oversizing can be overly conservative. This paper seeks to eliminate this conservatism by developing reference governor-based algorithms that enable smaller-sized batteries whose states satisfy operating constraints that explicitly model degradation mechanisms. This is in contrast to the traditional approach, which utilizes voltage and current limits that do not directly correspond to the internal degradation mechanisms.

A reference governor $(R G)$ is an effective tool for controlling a system within pointwise-in-time constraints. This add-on control scheme attenuates the command signal (electric current, in our case) to a system such that state constraints are satisfied while maintaining tracking performance [1]-[3]. This method has been applied to a variety of systems, including

H. E. Perez and S. J. Moura are with the Department of Civil and Environmental Engineering, and N. Shahmohammadhamedani is with the Department of Mechanical Engineering, University of California, Berkeley, CA 94720, USA (E-mail: \{heperez, niloofar, smoura\}@ berkeley.edu

${ }^{1} \mathrm{C}$-rate is a normalized measure of electric current that enables comparison between different sized batteries. It is defined as the ratio of current in Amperes (A) to a cell's nominal capacity in Ampere-hours (Ah). electrochemical energy conversation devices. For example, Sun and Kolmanovsky developed a robust nonlinear RG to protect against oxygen starvation in fuel cell systems [4]. In [5], Vahidi et al. adopted a so-called "Fast" RG approach for fuel cells to protect against compressor surge/chock and oxygen starvation. In battery systems, Plett designed an algorithm to determine power limits in real-time [6]. This approach considers an equivalent circuit model and terminal voltage constraints. Smith et al. utilized a reduced-order, linearized electrochemical model for state estimation and prediction of maximum, safe current draw [7]. Klein et al. use a detailed electrochemical model with nonlinear model predictive control to determine optimal charging trajectories subject to state constraints [8]. Hu et al. use equivalent circuit battery models to optimize charge time and power loss subject to state of charge, current, voltage, and charge time constraints [9].

In this paper we design schemes that govern commanded electrical current, in the presence of constraints on the electrochemical states. As such, this article's main contribution is the design of modified RGs for battery constraint management via electrochemical models. We present nonlinear and linear designs that trade-off guaranteed constraint satisfaction with computational efficiency. This article extends our previous work [10] with a comprehensive numerical study that quantifies the potential performance benefits of a modified RG over traditional voltage-based control, with respect to power, energy, and safety.

The remainder of this paper is structured as follows. Section II summarizes the electrochemical model and presents two motivating examples. Section III develops the nonlinear and linearized modified RGs. Section IV presents results using multiple drive cycles. Section V summarizes the main results.

\section{Electrochemical Model \& Motivation}

\section{A. Doyle-Fuller-Newman Model}

We consider the Doyle-Fuller-Newman (DFN) model in Fig. 1 to predict the evolution of lithium concentration in the solid $c_{s}^{ \pm}(x, r, t)$, lithium concentration in the electrolyte $c_{e}(x, t)$, solid electric potential $\phi_{s}^{ \pm}(x, t)$, electrolyte electric potential $\phi_{e}(x, t)$, ionic current $i_{e}^{ \pm}(x, t)$, molar ion fluxes $j_{n}^{ \pm}(x, t)$, and bulk cell temperature $T(t)$ [11]. The governing equations are

$$
\begin{aligned}
\frac{\partial c_{s}^{ \pm}}{\partial t}(x, r, t) & =\frac{1}{r^{2}} \frac{\partial}{\partial r}\left[D_{s}^{ \pm} r^{2} \frac{\partial c_{s}^{ \pm}}{\partial r}(x, r, t)\right], \\
\varepsilon_{e} \frac{\partial c_{e}}{\partial t}(x, t) & =\frac{\partial}{\partial x}\left[\varepsilon_{e} D_{e} \frac{\partial c_{e}}{\partial x}(x, t)+\frac{1-t_{c}^{0}}{F} i_{e}^{ \pm}(x, t)\right], \\
\frac{\partial \phi_{s}^{ \pm}}{\partial x}(x, t) & =\frac{i_{e}^{ \pm}(x, t)-I(t)}{\sigma^{ \pm}}, \\
\frac{\partial \phi_{e}}{\partial x}(x, t) & =-\frac{i_{e}^{ \pm}(x, t)}{\kappa}+\frac{2 R T}{F}\left(1-t_{c}^{0}\right)
\end{aligned}
$$




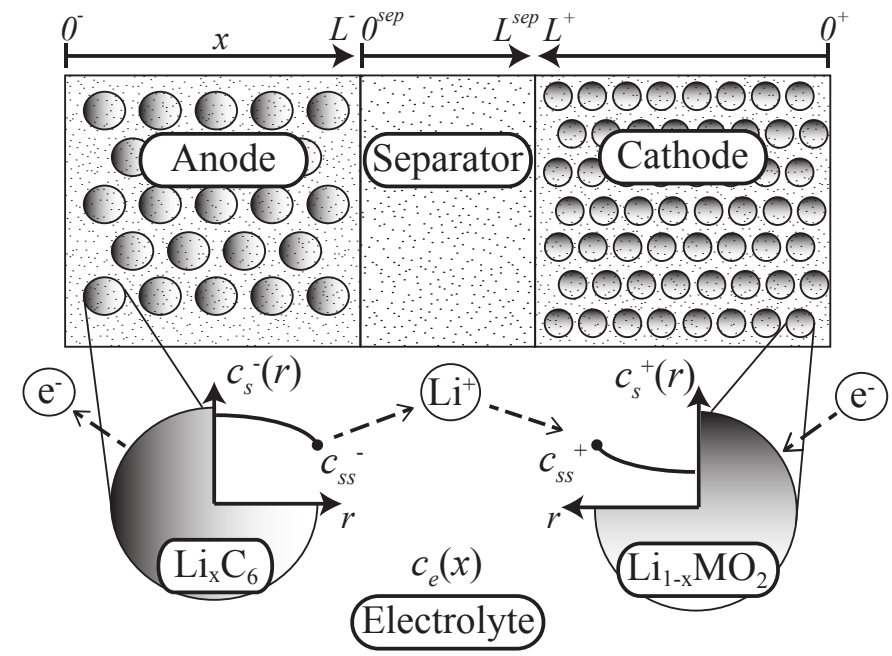

Fig. 1. Schematic of the Doyle-Fuller-Newman model [11]. The model considers two phases: the solid and electrolyte. In the solid, states evolve in the $x$ and $r$ dimensions. In the electrolyte, states evolve in the $x$ dimension only. The cell is divided into three regions: anode, separator, and cathode.

$$
\begin{aligned}
& \times\left(1+\frac{d \ln f_{c / a}}{d \ln c_{e}}(x, t)\right) \frac{\partial \ln c_{e}}{\partial x}(x, t) \\
\frac{\partial i_{e}^{ \pm}}{\partial x}(x, t) & =a_{s} F j_{n}^{ \pm}(x, t) \\
j_{n}^{ \pm}(x, t) & =\frac{1}{F} i_{0}^{ \pm}(x, t)\left[e^{\frac{\alpha_{a} F}{R T} \eta^{ \pm}(x, t)}-e^{-\frac{\alpha_{c} F}{R T} \eta^{ \pm}(x, t)}\right] \\
\rho^{\mathrm{avg}} c_{P} \frac{d T}{d t}(t) & =h_{\mathrm{cell}}\left[T_{\mathrm{amb}}(t)-T(t)\right]+I(t) V(t) \\
& -\int_{0^{-}}^{0^{+}} a_{s} F j_{n}(x, t) \Delta T(x, t) d x
\end{aligned}
$$

where $D_{e}, \kappa, f_{c / a}$ are functions of $c_{e}(x, t)$ and

$$
\begin{aligned}
i_{0}^{ \pm}(x, t)= & k^{ \pm}\left[c_{s s}^{ \pm}(x, t)\right]^{\alpha_{c}}\left[c_{e}(x, t)\left(c_{s, \max }^{ \pm}-c_{s s}^{ \pm}(x, t)\right)\right]^{\alpha_{a}} \\
\eta^{ \pm}(x, t)= & \phi_{s}^{ \pm}(x, t)-\phi_{e}(x, t) \\
& -U^{ \pm}\left(c_{s s}^{ \pm}(x, t)\right)-F R_{f}^{ \pm} j_{n}^{ \pm}(x, t), \\
c_{s s}^{ \pm}(x, t)= & c_{s}^{ \pm}\left(x, R_{s}^{ \pm}, t\right) \\
\Delta T(x, t)= & U^{ \pm}\left(\bar{c}_{s}^{ \pm}(x, t)\right)-T(t) \frac{\partial U^{ \pm}}{\partial T}\left(\bar{c}_{s}^{ \pm}(x, t)\right) \\
\bar{c}_{s}^{ \pm}(x, t)= & \frac{3}{\left(R_{s}^{ \pm}\right)^{3}} \int_{0}^{R_{s}^{ \pm}} r^{2} c_{s}^{ \pm}(x, r, t) d r
\end{aligned}
$$

Along with these equations are corresponding boundary and initial conditions. For brevity, we only summarize the differential equations here. Further details, including notation definitions, can be found in [11], [12]. The parameters are taken from the publicly available DUALFOIL model, developed by Newman and his collaborators [13]. The simulations provided here correspond to a $\mathrm{LiCoO}_{2}-\mathrm{C}$ cell. The cell capacity is $67 \mathrm{Ah} / \mathrm{m}^{2}$, calculated from the maximum concentration of the anode. However, the techniques are broadly applicable to any Li-ion chemistry.

\section{B. Constraints}

It is critical to maintain the battery within a safe operating regime. This protects against failure and maintains longevity. Towards this end, we consider several constraints,

$$
\begin{aligned}
\theta_{\min }^{ \pm} & \leq \frac{c_{s}^{ \pm}(x, r, t)}{c_{s, \max }^{ \pm}} \leq \theta_{\max }^{ \pm}, \\
c_{e, \min } & \leq c_{e}(x, t) \leq c_{e, \max }, \\
T_{\min } & \leq T(t) \leq T_{\max }, \\
\eta_{s}(x, t) & =\phi_{s}(x, t)-\phi_{e}(x, t)-U_{s} \geq 0 .
\end{aligned}
$$

Equations (13) and (14) protect the solid active material and electrolyte, respectively, from lithium depletion/oversaturation. Equation (15) protects against excessively cold or hot temperatures, which accelerates cell aging. Finally, (16) is a side reaction overpotential constraint. It models when unwanted side reactions occur, such as lithium plating [14], [15] when $U_{s}=0 \mathrm{~V}$ [12], and can also model accelerated growth of the solid/electrolyte interphase film formation [16], [17] when $U_{s}=0.4 \mathrm{~V}$ [17], [18].

\section{Numerical Implementation}

Numerical solution of the coupled nonlinear PDAE (1)-(12) is by itself a nontrivial task. A rich body of literature exists on this singular topic (cf. Ch. 4 of [19] and references therein). In our work the PDEs governing diffusion in the solid phase, (1), are discretized in the $r$-dimension via Padé approximates [20]. All the remaining PDEs are discretized in the $x$ dimension via the central difference method, such that the moles of lithium are conserved. This ultimately produces a finite-dimensional continuous-time differential-algebraic equation (DAE) system

$$
\begin{aligned}
\dot{x}(t) & =f(x(t), z(t), I(t)), \\
0 & =g(x(t), z(t), I(t)),
\end{aligned}
$$

where $x=\left[c_{s}^{ \pm}, c_{e}, T\right]^{T}, \quad z=\left[\phi_{s}^{ \pm}, i_{e}^{ \pm}, \phi_{e}, j_{n}^{ \pm}\right]^{T}$. This DAE model is then propagated forward in time via an implicit numerical scheme. In particular, the nonlinear discretized equations are solved via Newton's method, at each time step. A crucial step is to provide the scheme with analytic expressions for the Jacobian, which ensures fast convergence and accurate simulations. These Jacobians are also used for the linearized modified reference governor design in Section III-B.

\section{Motivating Examples}

Next, we consider two motivating examples: Li plating and Li depletion in the electrolyte. In Fig. 2 we consider a $10 \mathrm{sec}$, $3 \mathrm{C}$ pulse charging cycle at $80 \%$ SOC as an example scenario when Li plating may occur. The solid lines in Fig. 2 display the side reaction overpotential response at the anode/separator interface, $\eta_{s}\left(L^{-}, t\right)$. Note that $\eta_{s}\left(L^{-}, t\right)<0$ over several time periods. This induces Li plating, leading to dendrite formation that may potentially short-circuit the electrodes.

Figure 3 displays responses for $10 \mathrm{sec}$, 7C pulse discharging cycle at $60 \%$ SOC. Under this scenario, Li is eventually depleted at the cathode/current collector interface, denoted by solid lines $c_{e}\left(0^{+}, t\right)$. The model stops and becomes invalid after $66 \mathrm{sec}$ when $c_{e}\left(0^{+}, t\right)<0$. 

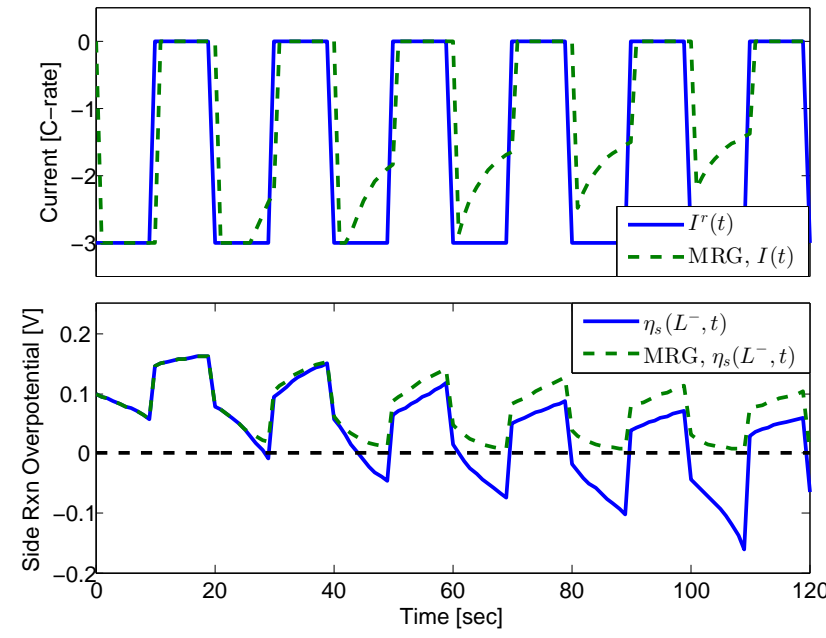

Fig. 2. Motivating example of Li plating. Evolution of current $I(t)$, reference current $I^{r}(t)$, and side reaction overpotential $\eta_{s}\left(L^{-}, t\right)$ for a $10 \mathrm{sec} 3 \mathrm{C}$ pulse charging scenario, with and without a modified reference governor.

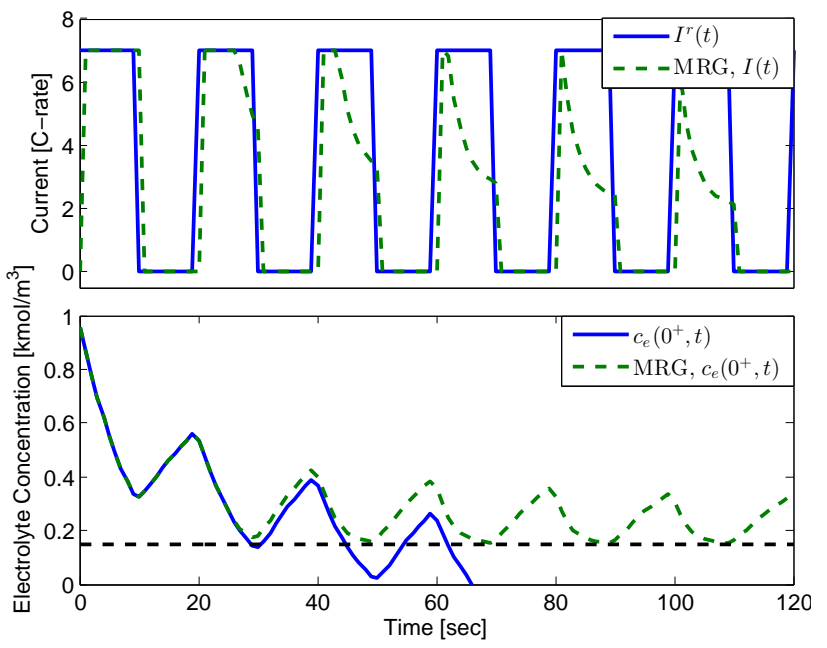

Fig. 3. Motivating example of lithium depletion in the electrolyte. The model is invalid after $c_{e}\left(0^{+}, t\right)<0$. Evolution of current $I(t)$, reference current $I^{r}(t)$, and electrolyte concentration $c_{e}\left(0^{+}, t\right)$ for a $10 \sec 7 \mathrm{C}$ pulse discharging scenario, with and without a modified reference governor.

In the following sections, we design an algorithm to protect the battery from entering these unsafe regions.

\section{Modified ReFEREnCE Governor (MRG) DESIGNS}

\section{A. Nonlinear MRG Design}

We utilize the RG concept to handle constraint satisfaction in batteries. A RG is an add-on system that guarantees constraint satisfaction and maintains a desired level of reference tracking. It operates in a discrete-time domain, since the computations may not be feasibly performed in real-time. In our "modified" RG approach, the applied current $I(t)$ and reference current $I^{r}(t)$ are related according to

$$
I[k+1]=\beta[k] I^{r}[k], \quad \beta \in[0,1],
$$

where $I(t)=I[k]$ for $t \in[k \Delta t,(k+1) \Delta t), k \in \mathbb{Z}$, and similarly for $I^{r}[k]$. We define the admissible set

$$
\mathcal{O}=\left\{(x(t), z(t)): y(\tau) \in \mathcal{Y}, \forall \tau \in\left[t, t+T_{s}\right]\right\}
$$

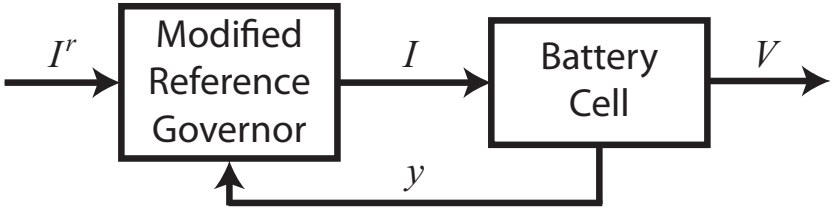

Fig. 4. Block diagram of modified reference governor with direct measurements of the constrained variables $y$.

where

$$
\begin{aligned}
\dot{x}(t) & =f\left(x(t), z(t), \beta I^{r}\right), \\
0 & =g\left(x(t), z(t), \beta I^{r}\right), \\
y(t) & =C_{1} x(t)+C_{2} z(t)+D \cdot \beta I^{r}+E .
\end{aligned}
$$

The output variables $y=\left[c_{s}^{ \pm}, c_{e}, T, \eta_{s}\right]^{T}$ must exist in set $\mathcal{Y}$, characterized by inequalities (13)-(16). The goal is to find the maximum value of $\beta$ which maintains the state in $\mathcal{O}$

$$
\beta^{*}[k]=\max \{\beta \in[0,1]:(x(t), z(t)) \in \mathcal{O}\},
$$

where $(x(t), z(t))$ depends on $\beta$ via (20)-(23).

To determine parameter $\beta^{*}$ at each time instant, the electrochemical model is simulated forward over the time interval $\left[t, t+T_{s}\right]$, where $T_{s}$ is the simulation horizon. If the constraints are violated for a given value of $\beta$, then $\beta$ is reduced and the model is re-simulated to ascertain constraint satisfaction of the new value of $\beta$. If the constraints are satisfied, then $\beta$ is increased to reduce tracking error between $I(t)$ and $I^{r}(t)$. This process is iterated according to the bisection algorithm.

Remark 1: We refer to (19) as a "modified" RG to distinguish it from the conventional RG concept that assumes an asymptotically stable system and applies input

$$
I[k+1]=I[k]+\beta[k]\left(I^{r}[k]-I[k]\right), \quad \beta \in[0,1],
$$

which inserts a low-pass filter between the reference and applied inputs [1], [2]. A battery is not asymptotically stable, but marginally stable. That is, an eigenvalue at the origin ensures conservation of lithium, which is the key energy storage property of batteries. Hence, we modify the conventional RG such that a zero current input is always feasible and returns the battery equilibrium. A similar concept is used in [7].

\section{B. Linear MRG Design}

The nonlinear MRG developed in the previous section achieves guaranteed constraint satisfaction at the expense of computational effort. Computational complexity, however, is often the deciding factor on which design ultimately reaches implementation. Next we design and evaluate a computationally efficient MRG based upon a linearized model. The critical benefit of the linear MRG is that the parameter $\beta$ can be determined by an explicit expression. In contrast, the nonlinear MRG requires simulations and optimization.

At each time step we linearize the model (21)-(22) around the state and input values from the previous time step: $\left(x^{0}, z^{0}, u^{0}\right)=(x[k-1], z[k-1], I[k-1])$ to obtain evolution equations

$$
\dot{\tilde{x}}=A_{11} \tilde{x}+A_{12} \tilde{z}+B_{1} \tilde{I}
$$




$$
0=A_{21} \tilde{x}+A_{22} \tilde{z}+B_{2} \tilde{I},
$$

where $\tilde{x}=x-x^{0}, \tilde{z}=z-z^{0}, \tilde{I}=\beta I^{r}-I^{0}$ and $A_{11}, A_{12}, A_{21}, A_{22}, B_{1}, B_{2}$ are the Jacobian terms of the nonlinear state equations (21)-(22), evaluated at $\left(x^{0}, z^{0}, u^{0}\right)$. Since this DAE system is linear and semi-explicit of index 1, we can explicitly solve for $\tilde{z}$ and write the system as

$$
\dot{\tilde{x}}=A \tilde{x}+B \tilde{I}
$$

where $A=A_{11}-A_{12} A_{22}^{-1} A_{21}$ and $B=B_{1}-A_{12} A_{22}^{-1} B_{2}$. Under this representation, the states after a simulation horizon horizon of $T_{s}$, can be computed analytically. That is,

$$
\begin{aligned}
& \tilde{x}\left(t+T_{s}\right)=e^{A T_{s}} \tilde{x}(t)+\int_{t}^{t+T_{s}} e^{A\left(t+T_{s}-\tau\right)} B \tilde{I} d \tau, \\
& \tilde{z}\left(t+T_{s}\right)=-A_{22}^{-1}\left[A_{21} \tilde{x}\left(t+T_{s}\right)+B_{2} \tilde{I}\right] .
\end{aligned}
$$

The constrained output variables after $T_{s}$ time units are

$$
\begin{aligned}
y\left(t+T_{s}\right)= & C_{1}\left[x^{0}+\tilde{x}\left(t+T_{s}\right)\right]+C_{2}\left[z^{0}+\tilde{z}\left(t+T_{s}\right)\right] \\
& +D \cdot \beta I^{r}+E \leq 0
\end{aligned}
$$

where $C_{1}, C_{2}, D, E$ are matrices which incorporate inequalities (13)-(16). We also assume the reference current $I^{r}$ is constant over the simulation horizon - a typical assumption in RG design [1], [2], [4], [5], [7]. We are now positioned to formulate the linearized MRG problem. Given the current states and reference current $\left(x(t), z(t), I^{r}(t)\right)$, solve

$$
\max _{\beta \in[0,1]} \beta, \quad \text { subject to } \beta F \leq G
$$

where $F, G$ are vectors that incorporate the constraints (13)-(16) and depend on $x(t)$ and $I^{r}(t)$ as follows

$$
\begin{aligned}
F= & {\left[C_{1} L-C_{2} A_{22}^{-1}\left(A_{21} L+B_{2}\right)+D\right] I^{r}, } \\
G= & -E-C_{1}\left[x^{0}+\Phi\left(x(t)-x^{0}\right)-L I^{0}\right] \\
& -C_{2}\left[z^{0}-A_{22}^{-1}\left[A_{21}\left(\Phi\left(x(t)-x^{0}\right)-B_{2} I^{0}\right]\right],\right.
\end{aligned}
$$

where

$$
\Phi=e^{A T_{s}}, \quad L=\int_{t}^{t+T_{s}} e^{A\left(t+T_{s}-\tau\right)} B d \tau .
$$

The optimization problem (32) is a one-dimensional linear program. Consequently, it can be solved explicitly by determining the dominating constraint

$$
\begin{aligned}
H_{i} & =\left\{\begin{array}{ll}
G_{i} / F_{i} & \text { if } \quad F_{i}>0 \\
-G_{i} / F_{i} & \text { else }
\end{array} \quad i=1,2, \ldots, N_{c},\right. \\
\beta^{*} & =\min \left\{1, H_{i} \quad \mid i=1,2, \ldots, N_{c}\right\},
\end{aligned}
$$

where $G_{i}$ and $F_{i}$ denote the $i^{\text {th }}$ element of $G$ and $F$, respectively, and $N_{c}$ is the total number of elements.

\section{NUMERICAL RESUlts}

1) MRG Simulations: We consider the case when the constrained output variables, $y$, are measurable, as shown in Fig. 4. In practice, one needs to estimate these variables from measurements of current and voltage, as done in [21]. This section analyzes performance under the hypothetical situation of output variable feedback. Prediction horizon $T_{s}=5 \mathrm{sec}$ is used in all simulations.

In the following, we apply the MRG to the scenarios described in Section II-D. Figure 2 displays the current $I(t)$, reference current $I^{r}(t)$, and side reaction overpotential $\eta_{s}\left(L^{-}, t\right)$ for a $10 \sec 3 \mathrm{C}$ pulse charging scenario. Note how the MRG attenuates the current to satisfy $\eta_{s}>0$. Similarly, Fig. 3 displays the system responses for a $10 \mathrm{sec} 7 \mathrm{C}$ pulse discharging scenario. Again, $I(t)$ is attenuated such that lithium is not depleted in the electrolyte.

Next we demonstrate the benefits of utilizing a MRG for charging. Figure 5 compares the standard charging protocol, constant charging-constant voltage (CCCV), to a reference governor-based charging. In both cases, we consider a constant $1 \mathrm{C}$ charging current. The CCCV protocol applies $1 \mathrm{C}$ charging until the terminal voltage reaches a "maximum safe voltage level," $4.2 \mathrm{~V}$ in this case. This occurs near the 7.5 min. mark. Then CCCV regulates terminal voltage at the maximum value, $4.2 \mathrm{~V}$, while the current diminishes toward zero. The $4.2 \mathrm{~V}$ limit is selected such that lithium plating does not occur due to overcharging. Indeed, the side reaction overpotential remains positive. However, this approach is conservative. Specifically, the side reaction overpotential can be regulated closer to its limit. The MRG applies 1C charging subject to the constraint $\eta_{s}\left(L^{-}, t\right) \geq 0$. In Fig. 5 the MRG maintains $\eta_{s} \geq 0$ despite voltage exceeding $4.2 \mathrm{~V}$. Moreover, the cell attains $95 \%$ SOC in $14.9 \mathrm{~min}$ using the MRG vs $35.5 \mathrm{~min}$ for CCCV. Also note that $\mathrm{CCCV}$ reaches an equilibrium SOC of $96 \%$, whereas the MRG achieves 100\% SOC. Consequently, 60\%-95\% charging time is decreased by $58 \%$ and charge capacity is increased by $4 \%$.

2) Linear-MRG Simulations: Next we evaluate simulations of the linear MRG (LMRG) to ascertain the trade off between computational efficiency and constraint satisfaction. Figure 6 compares the LMRG to the nonlinear MRG, for the 10sec 3C pulse charging scenario. In the LMRG, $\eta_{s}\left(L^{-}, t\right)$ does not reach the constraint, due to linearization modeling errors. This produces a conservative response that is within the constraint. The opposite is portrayed in Fig. 7, for the 10sec 7C pulse discharging scenario, where $c_{e}\left(0^{+}, t\right)$ violates the constraint over several time periods. One might interpret the constraint over/undershoot as follows. All the constraints can be categorized into "soft constraints" (small violations are allowable but undesirable, e.g. SEI film growth) and "hard constraints" (small violations are not allowable, e.g. electrolyte depletion). For hard constraints, the limits can be selected more conservatively to avoid overshoots. Nonetheless, the constraint violation magnitude is relatively small and the LMRG would be effective at mitigating degradation and prolonging battery life.

The critical advantage of the LMRG, however, is the in-

TABLE I

CPU TIME PER Simulated Time For Nonlinear and Linear MRGs.

\begin{tabular}{c|c|c}
\hline \hline Scenario & MRG & Linear MRG \\
\hline 10sec 3C charging & $1.48 \mathrm{sec} / \mathrm{sec}(100 \%)$ & $0.34 \mathrm{sec} / \mathrm{sec}(23 \%)$ \\
10sec 7C discharging & $2.16 \mathrm{sec} / \mathrm{sec}(100 \%)$ & $0.39 \mathrm{sec} / \mathrm{sec}(18 \%)$ \\
\hline \hline
\end{tabular}




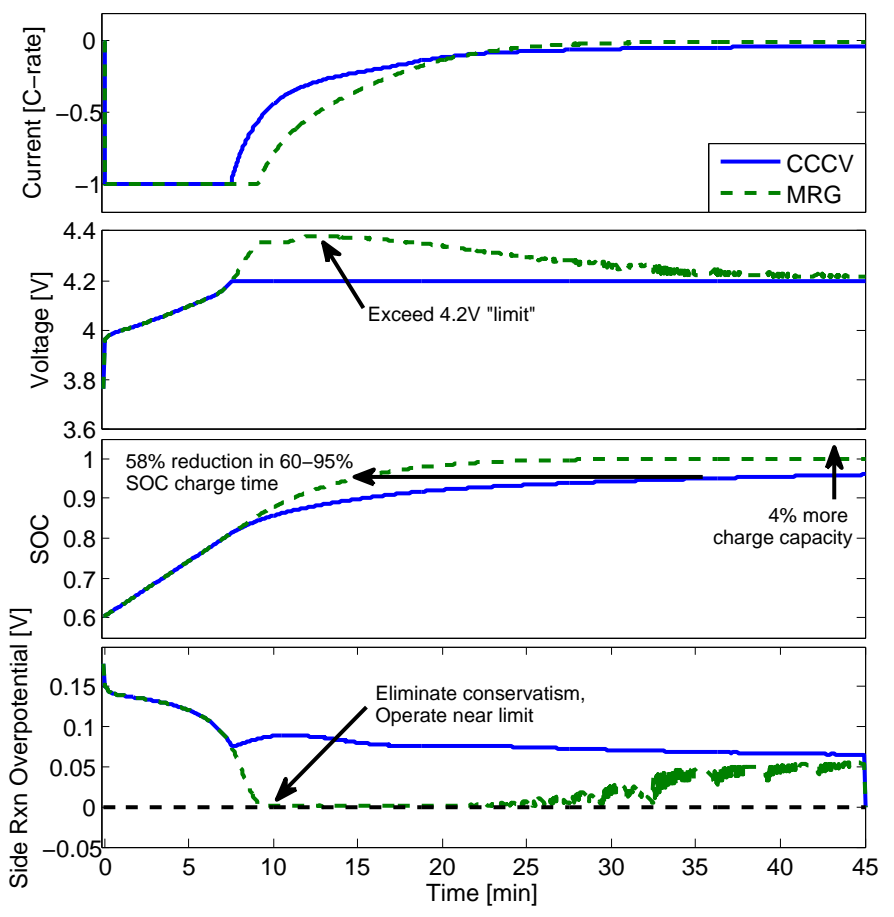

Fig. 5. Comparison of $\mathrm{CCCV}$ and modified reference governor (MRG) charging. The MRG regulates $\eta_{s}$ near its limit, thereby achieving $95 \%$ SOC in $14.9 \mathrm{~min}$ vs. $35.5 \mathrm{~min}$ for CCCV by allowing voltage to safely exceed $4.2 \mathrm{~V}$.

creased computational efficiency. That is, the LMRG computes $\beta$ via the explicit expressions (33)-(37), whereas the nonlinear MRG requires nonlinear simulations and optimization. We consider the CPU time for each MRG as one measure of computational efficiency. The data provided in Table I indicates that the linear MRG reduces CPU time by over four-fold on a $2.9 \mathrm{GHz}$ dual-core laptop with $16 \mathrm{~GB}$ of RAM. Further improvements are possible via code optimization.

Remark 2 (Current Limits \& Power Capacity): The LMRG also provides real-time estimates of the $\max / \mathrm{min}$ safe current and power capacity. The limiting current is given by

$$
I_{\lim }(t)=I^{r}(t) \cdot \min \left\{H_{i} \mid i=1,2, \ldots, N_{c}\right\},
$$

and the corresponding instantaneous power capacity is

$$
P_{\text {cap }}(t)=I_{\text {lim }}(t) V(t) .
$$

These variables are useful for feedback to higher-level supervisory control systems [6], [7], [21].

\section{A. Comparative Analysis}

We evaluate the operational, power and energy capacity benefits of the MRG versus an industry standard Voltage-Only (VO) controller on electric vehicle-like charge/discharge cycles. For comparison purposes, we choose operational voltage limits of $2.8 \mathrm{~V}$ and $3.9 \mathrm{~V}$ for the $\mathrm{VO}$ controller. Various automotiverelevant charge/discharge cycles cases were tested. To explore state constraint management, reference current was scaled by factors of $\times 1.0, \times 1.2, \times 1.4(1.0 \mathrm{I}, 1.2 \mathrm{I}, 1.4 \mathrm{I})$. The $\mathrm{MRG}$ constraints from (13) - (16) chosen for this analysis are the: Surface Concentrations $\theta\left(0^{-}, t\right), \theta\left(L^{-}, t\right), \theta\left(0^{+}, t\right), \theta\left(L^{+}, t\right)$, Electrolyte Concentration $c_{e}\left(0^{+}, t\right), c_{e}\left(0^{-}, t\right)$, Temperature
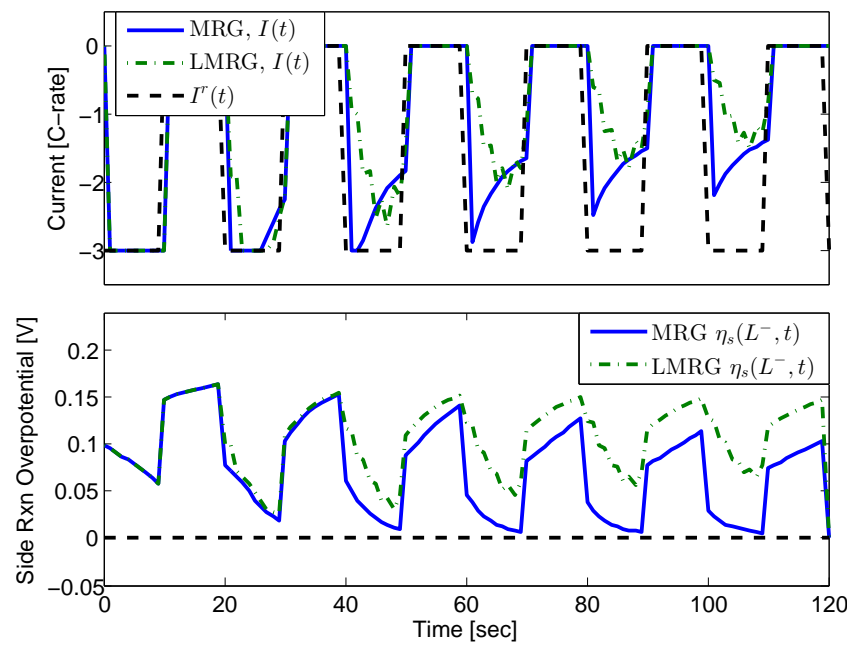

Fig. 6. Comparison of MRG and LMRG. Signals include current $I(t)$, reference current $I^{r}(t)$, and side reaction overpotential $\eta_{s}\left(L^{-}, t\right)$ for a $10 \mathrm{sec}$ $3 \mathrm{C}$ pulse charging scenario. The LMRG does not reach the constraint, due to linearization modeling errors.

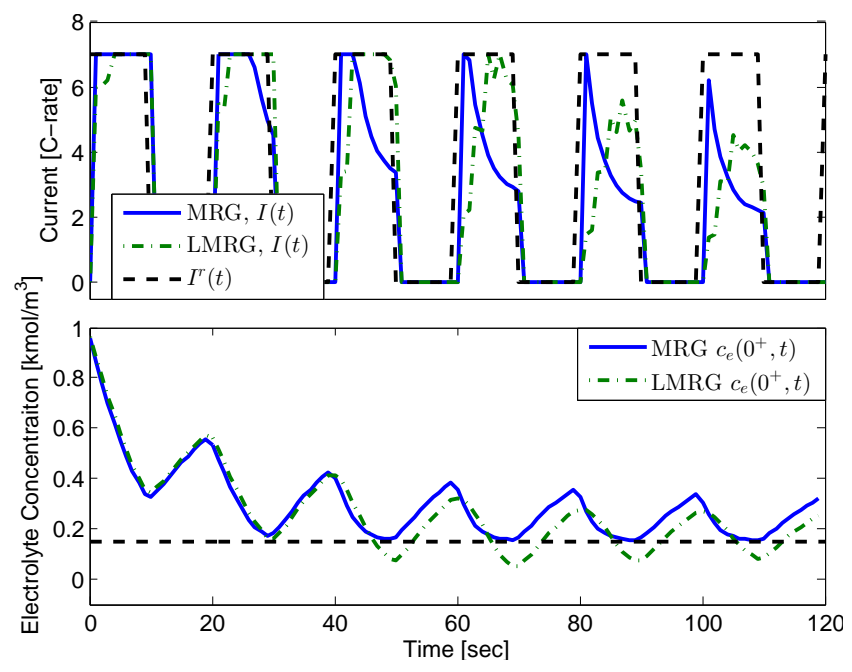

Fig. 7. Comparison of MRG and LMRG. Signals include current $I(t)$, reference current $I^{r}(t)$, and electrolyte concentration $c_{e}\left(0^{+}, t\right)$ for a $10 \mathrm{sec}$ 7C pulse discharging scenario. The LMRG violates the constraint, due to linearization modeling errors.

$T(t)$, and Side Reaction Overpotential $\eta_{s}\left(L^{-}, t\right)$. The constraint regions represent critical locations where the variable is most likely to be largest/smallest, respectively, for upper/lower bounds. It is assumed that that $U_{s}=0$ for the Side Reaction Overpotential $\eta_{s}\left(L^{-}, t\right)$, and hence are constraining Li plating from occurring. Due to space constraints, we only provide detailed examples with three concatenated US06 drive cycles (US06x3).

Figure 8 shows simulation results for the US06x3 profile whose current is scaled up by $40 \%$ (1.4I), applied to the VO controller. The upper voltage limit is first regulated before the 1 min mark, while the electrochemical variables are still away from their limits. One could operate the battery safely beyond this maximum voltage. Additionally, electrolyte concentration at the cathode/current collector interface $c_{e}\left(0^{+}, t\right)$ falls below its lower limit near $10 \mathrm{~min}$, which induces Li plating. 

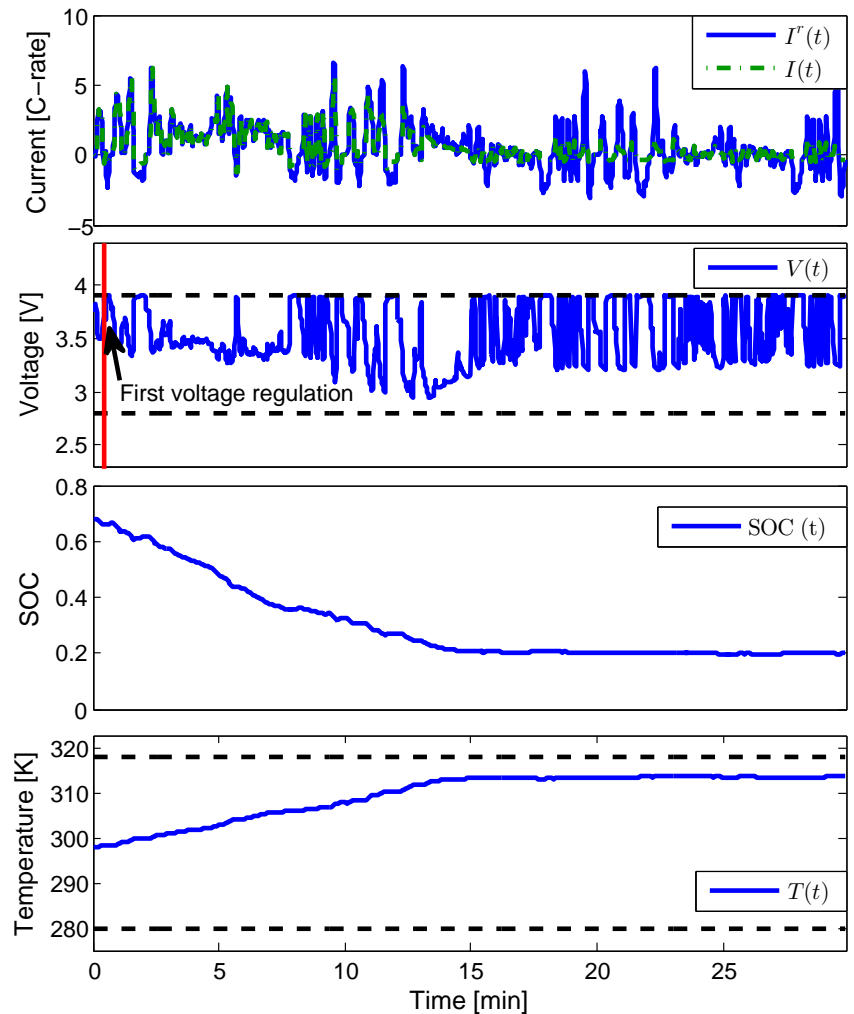
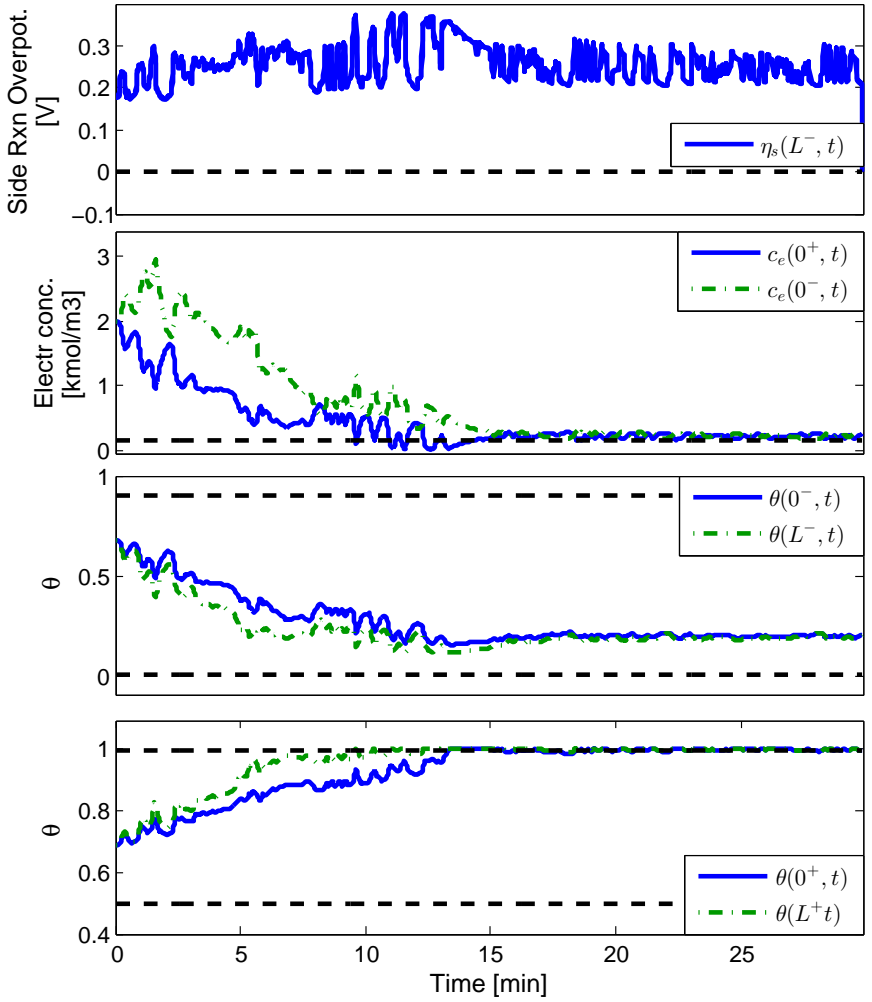

Fig. 8. US06x3 1.4I VO: Left: Reference Current $I^{r}(t)$ and Current $I(t)$, Voltage $V(t)$, State of Charge $S O C(t)$, Temperature $T(t)$. Right: Side Reaction Overpotential $\eta_{s}\left(L^{-}, t\right)$, Electrolyte Concentration $c_{e}\left(0^{+}, t\right), c_{e}\left(0^{-}, t\right)$, Surface Concentrations $\theta\left(0^{-}, t\right), \theta\left(L^{-}, t\right), \theta\left(0^{+}, t\right), \theta\left(L^{+}, t\right)$.
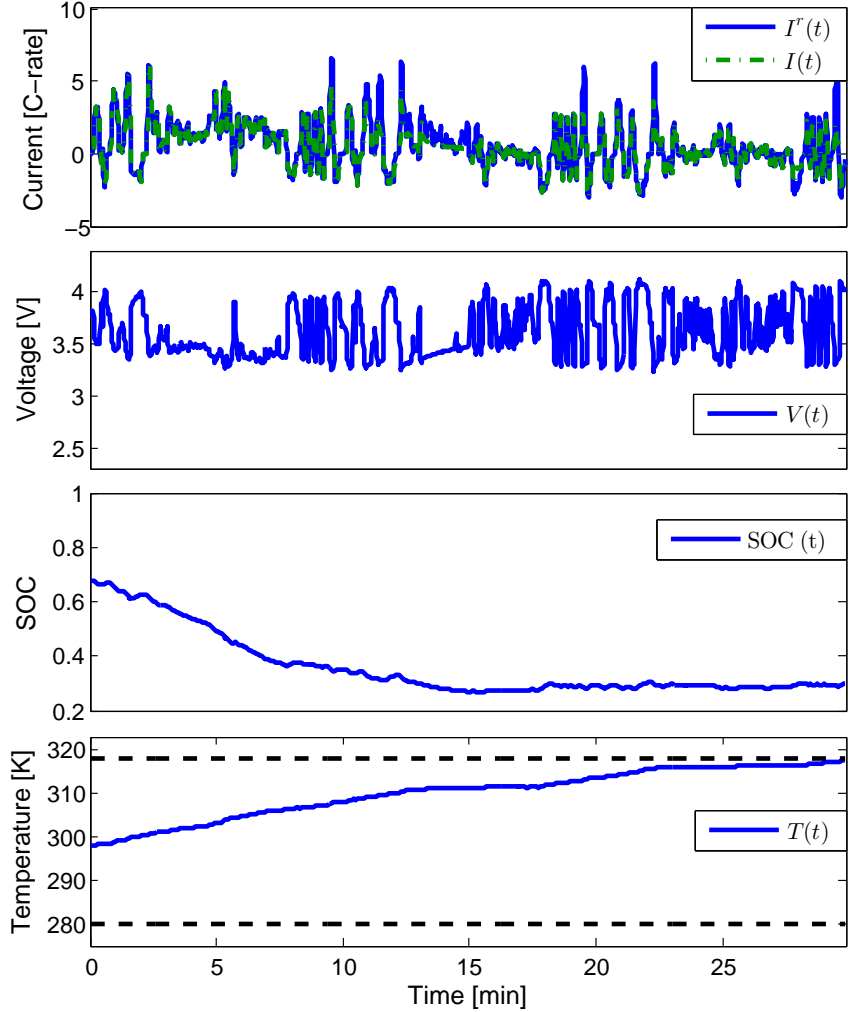
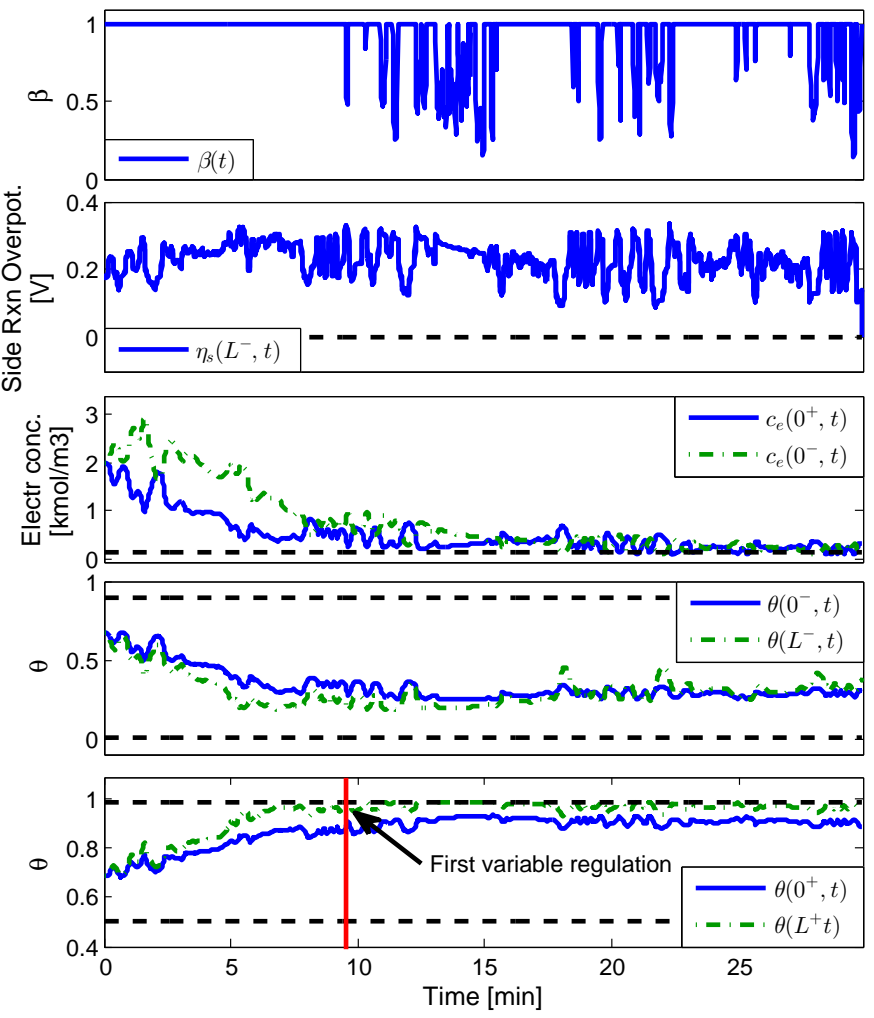

Fig. 9. US06x3 (1.4I) MRG. Left: Reference Current $I^{r}(t)$ and Current $I(t)$, Voltage $V(t)$, State of Charge $S O C(t)$, Temperature $T(t)$. Right: $\beta(t)$, Side Reaction Overpotential $\eta_{s}\left(L^{-}, t\right)$, Electrolyte Concentration $c_{e}\left(0^{+}, t\right), c_{e}\left(0^{-}, t\right)$, Surface Concentrations $\theta\left(0^{-}, t\right), \theta\left(L^{-}, t\right), \theta\left(0^{+}, t\right), \theta\left(L^{+}, t\right)$. 

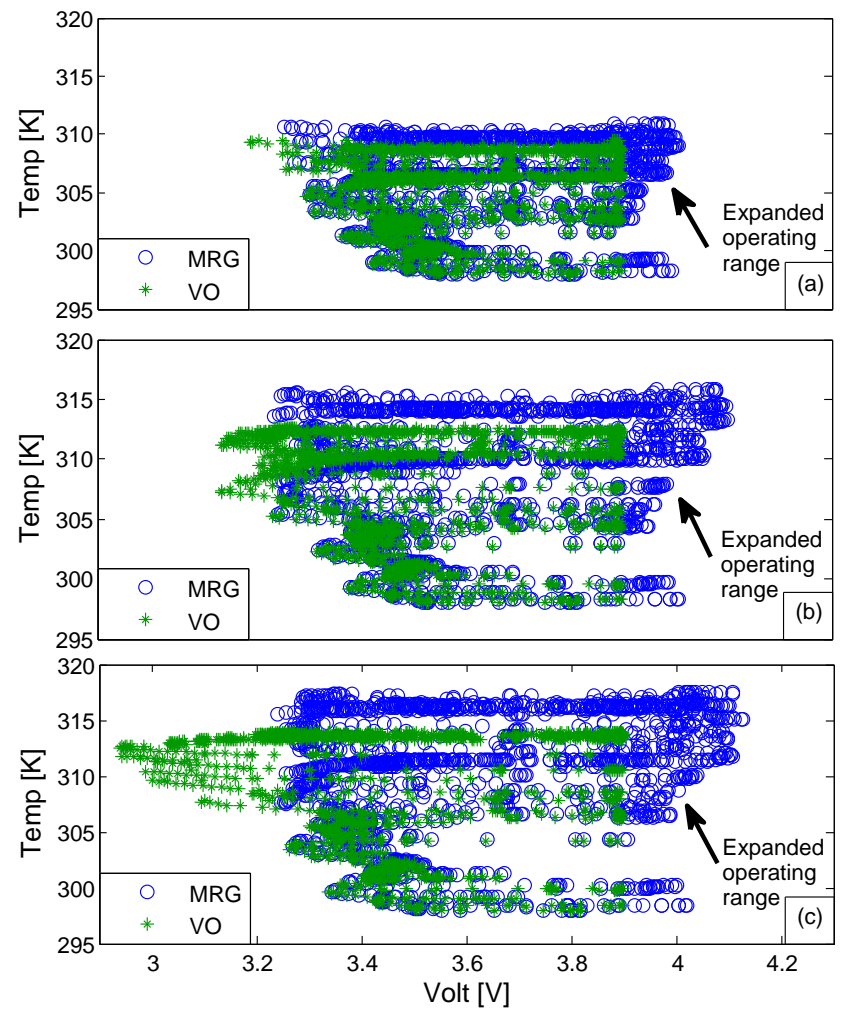

Fig. 10. Temperature vs. Voltage operating points for (a) 1.0I, (b) 1.2I, and (c) $1.4 \mathrm{I}$ over US06x3 cycle.

Figure 9 shows the simulation results for the US06x3 profile whose current is scaled up by $40 \%$ (1.4I), applied to the MRG controller. Note that the maximum $\mathrm{Li}$ concentration at the cathode/separator interface $\theta\left(L^{+}, t\right)$ limit is first regulated around the 9 min mark, yet the voltage exceeds the VO upper voltage limit before $9 \mathrm{~min}$. All other constrained electrochemical states are maintained within safe limits. This expands the operating regime, safely.

1) Expanded Operating Regime: Figure 10 depicts the Temperature vs. Voltage operational points for the MRG vs. VO controllers for the US06x3 1.0I, 1.2I, and 1.4I current profiles. The upper voltage limit on the VO controller becomes more constrictive as the current magnitude is scaled up. The MRG safely exceeds the VO voltage limits under all conditions, as previously noted. In automotive applications, this ultimately means the MRG is able to recuperate more energy (i.e. from regenerate braking) than the VO controller.

2) Increased Power Capacity: Figure 11 exemplifies how the MRG allows increased power capacity. It provides power responses for the MRG vs. VO controller for US06x3 1.0I, 1.2I, and 1.4I current profiles. As current is increased, the $\mathrm{VO}$ attenuates power to respect the voltage limits, whereas the MRG allows for increased power. Figure 12 displays the distribution of cell power for the MRG vs. VO controller. This distribution elucidates how the MRG allows for greater charge power (negative power) than the VO controller.

Table II presents the mean power (discharge and charge) benefit percentage results from using the MRG over the VO controller for the US06x3 drive cycle as well as five other automotive drive cycles (UDDSx2, SC04x4, LA92x2, DC1,
TABLE II

MEAN POWER BENEFITS OF USING MRG VS. VO.

\begin{tabular}{c|c|c|c|c}
\hline \hline Drive Cycle & Mode & $1.0 \mathrm{I}$ & $1.2 \mathrm{I}$ & $1.4 \mathrm{I}$ \\
\hline DC1 & Discharge & $0.09 \%$ & $0.24 \%$ & $4.02 \%$ \\
& Charge & $6.17 \%$ & $13.23 \%$ & $21.21 \%$ \\
\hline DC2 & Discharge & $0.02 \%$ & $-0.21 \%$ & $-0.75 \%$ \\
& Charge & $6.50 \%$ & $22.57 \%$ & $40.38 \%$ \\
\hline LA92x2 & Discharge & $0.09 \%$ & $1.79 \%$ & $8.91 \%$ \\
& Charge & $16.66 \%$ & $36.11 \%$ & $58.07 \%$ \\
\hline SC04x4 & Discharge & $0.08 \%$ & $0.18 \%$ & $2.97 \%$ \\
& Charge & $15.71 \%$ & $26.17 \%$ & $39.11 \%$ \\
\hline UDDSx2 & Discharge & $0.04 \%$ & $0.18 \%$ & $3.07 \%$ \\
& Charge & $6.02 \%$ & $20.09 \%$ & $33.49 \%$ \\
\hline US06x3 & Discharge & $0.23 \%$ & $5.60 \%$ & $11.33 \%$ \\
& Charge & $44.56 \%$ & $100.38 \%$ & $150.61 \%$ \\
\hline Average & Discharge & $0.09 \%$ & $1.29 \%$ & $4.92 \%$ \\
& Charge & $15.94 \%$ & $36.43 \%$ & $57.15 \%$ \\
\hline Std. Dev. & Discharge & $0.07 \%$ & $2.03 \%$ & $4.02 \%$ \\
& Charge & $13.56 \%$ & $29.42 \%$ & $43.19 \%$ \\
\hline \hline
\end{tabular}

TABLE III

ENERGY BENEFITS OF USING MRG VS. VO.

\begin{tabular}{c|c|c|c}
\hline \hline Drive Cycle & $1.0 \mathrm{I}$ & $1.2 \mathrm{I}$ & $1.4 \mathrm{I}$ \\
\hline DC1 & $2.77 \%$ & $5.59 \%$ & $4.68 \%$ \\
DC2 & $1.06 \%$ & $3.56 \%$ & $7.64 \%$ \\
LA92x2 & $7.25 \%$ & $11.95 \%$ & $10.65 \%$ \\
SC04x4 & $4.29 \%$ & $6.69 \%$ & $7.34 \%$ \\
UDDSx2 & $1.95 \%$ & $5.71 \%$ & $6.94 \%$ \\
US06x3 & $15.34 \%$ & $20.64 \%$ & $22.99 \%$ \\
\hline Average & $5.45 \%$ & $9.02 \%$ & $10.04 \%$ \\
Std. Dev. & $4.84 \%$ & $5.79 \%$ & $6.05 \%$ \\
\hline \hline
\end{tabular}

DC2) from [16]. In the most aggressive drive cycle (US06x3) the MRG achieves $11.03 \%$ and $150.61 \%$ more discharge and charge power, respectively, over the VO controller in the 1.4I case. Across all six simulated drive cycles, the MRG achieves average increases in discharge and charge power of $4.92 \%$ and $57.15 \%$, with a standard deviation of $4.02 \%$ and $43.19 \%$, respectively, in the $1.4 \mathrm{I}$ case.

3) Increased Energy Capacity: Table III presents the net energy benefits for six drive cycles (US06x3, UDDSx2, SC04x4, LA92x2, DC1, DC2). In the most aggressive drive cycle (US06x3) the MRG achieves a 22.99\% net energy increase over the VO controller for the $1.4 \mathrm{I}$ case. Across all six simulated drive cycles, the MRG achieves an average net energy increase of $10.04 \%$ with a standard deviation of $6.05 \%$ in the $1.4 \mathrm{I}$ case.

\section{CONCLUSIONS}

This paper develops reference governor-based approaches to satisfying electrochemical state constraints in batteries. As a consequence, it enables one to enhance power capacity, energy capacity, and charging speed by eliminating the conservatism imposed by traditional operating constraints (e.g. voltage limits). The key ingredients to this approach are the following. First, we utilize a first principles electrochemical model to predict and 

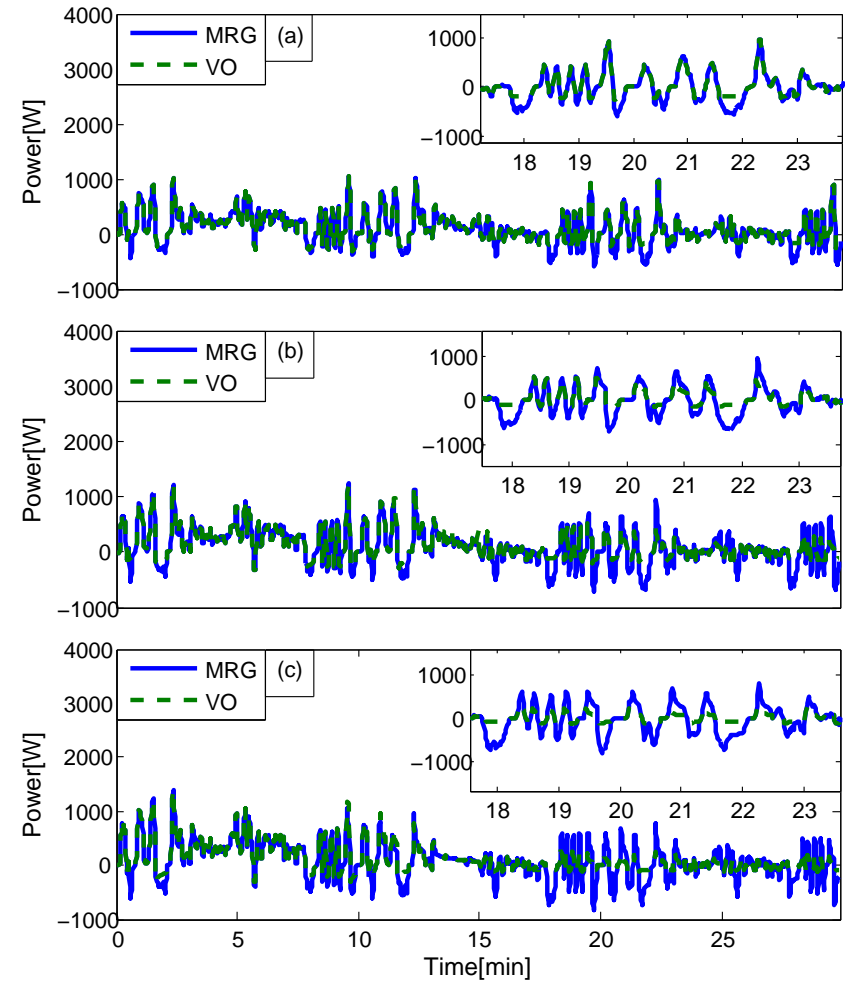

Fig. 11. US06x3 power responses for (a) 1.0I, (b) 1.2I, and (c) 1.4I.

constrain the evolution of physical degradation mechanisms. Second, a nonlinear modified reference governor (MRG) algorithm is developed assuming measurements of the constrained variables. Third, a linearized MRG is developed, which replaces simulations with an explicit function evaluation at the expense of possible constraint dissatisfaction or conservatism. A suite of simulations were executed to quantify the potential performance gains of MRGs over voltage-only regulators. We found 60\%$95 \%$ charge times can be reduced by $58 \%$, charge power can be increased by $57.15 \%$ on average, and energy can be increased by $10.04 \%$ on average, for the considered case studies.

In this paper we assume full state measurements and known parameters to ascertain the maximum possible performance benefits of MRG-based control. Future work combines the MRGs developed here with state and parameter estimates generated by adaptive PDE observers [21]. This outputfeedback system (i) guards against harmful operating regimes, (ii) increases energy capacity, power capacity, and charging speed, and (iii) monitors state-of-charge and state-of-health, all from measurements of voltage, current, and temperature. Finally, we plan to experimentally quantify the aforementioned benefits on a battery-in-the-loop test facility.

\section{APPENDIX}

\section{REFERENCES}

[1] E. Gilbert, I. Kolmanovsky, and K. Tan, "Discrete-time reference governors and the nonlinear control of systems with state and control constraints," International Journal of Robust and Nonlinear Control, vol. 5, no. 5, pp. 487-504, 1995.

[2] A. Bemporad, "Reference governor for constrained nonlinear systems," IEEE Trans. on Automatic Control, vol. 43, no. 3, pp. 415-419, 1998.
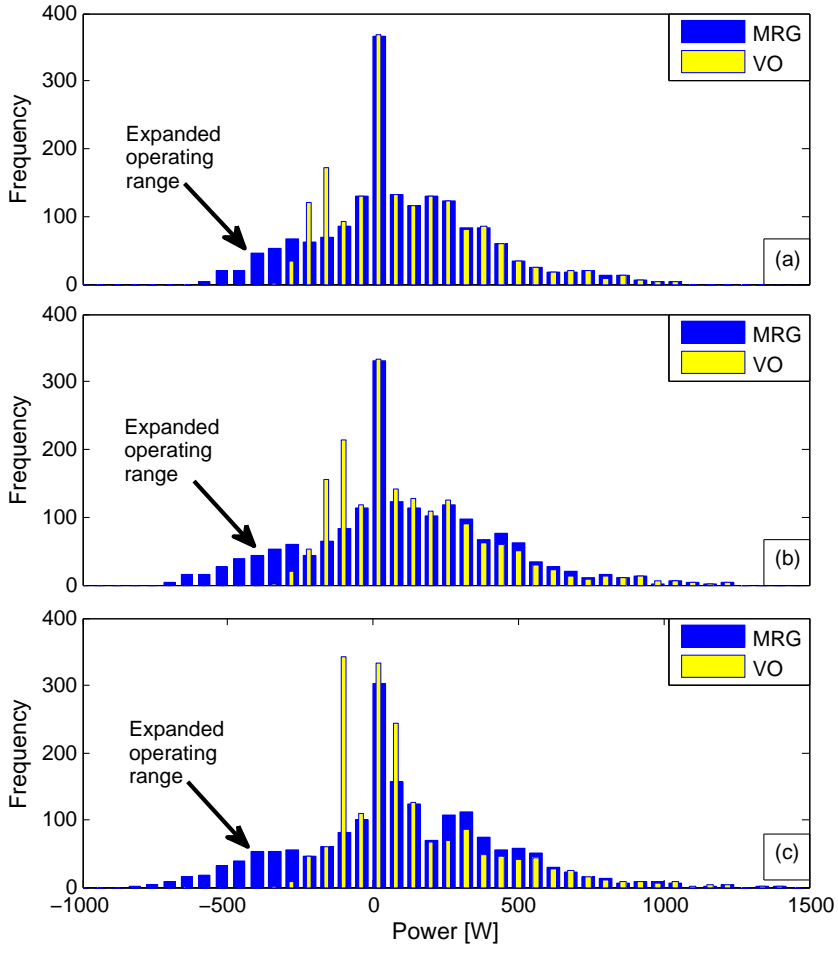

Fig. 12. US06x3 Power Histogram for (a) 1.0I, (b) 1.2I, and (c) 1.4I.

[3] I. Kolmanovsky, E. Garone, and S. Di Cairano, "Reference and command governors: A tutorial on their theory and automotive applications," in American Control Conference (ACC), 2014, June 2014, pp. 226-241.

[4] J. Sun and I. V. Kolmanovsky, "Load governor for fuel cell oxygen starvation protection: A robust nonlinear reference governor approach," IEEE Trans. on Control Systems Technology, vol. 13, no. 6, pp. 911 920, 2005.

[5] A. Vahidi, I. Kolmanovsky, and A. Stefanopoulou, "Constraint handling in a fuel cell system: A fast reference governor approach," IEEE Trans. on Control Systems Technology, vol. 15, no. 1, pp. 86-98, 2007.

[6] G. Plett, "High-performance battery-pack power estimation using a dynamic cell model," IEEE Trans. on Vehicular Technology, vol. 53, no. 5 , pp. 1586 - 1593, Sept. 2004.

[7] K. A. Smith, C. D. Rahn, and C.-Y. Wang, "Model-based electrochemical estimation and constraint management for pulse operation of lithium ion batteries," IEEE Trans. on Control Systems Technology, vol. 18, no. 3, pp. $654-663,2010$.

[8] R. Klein, N. A. Chaturvedi, J. Christensen, J. Ahmed, R. Findeisen, and A. Kojic, "Optimal charging strategies in lithium-ion battery," in American Control Conference, San Francisco, CA, United states, 2011, pp. $382-387$.

[9] X. Hu, S. Li, H. Peng, and F. Sun, "Charging time and loss optimization for linme and lifepo4 batteries based on equivalent circuit models," Journal of Power Sources, vol. 239, no. 0, pp. 449 - 457, 2013.

[10] S. Moura, N. Chaturvedi, and M. Krstic, "Constraint management in Li-ion batteries: A modified reference governor approach," in American Control Conference (ACC), 2013. IEEE, 2013, pp. 5332-5337.

[11] K. Thomas, J. Newman, and R. Darling, Advances in Lithium-Ion Batteries. New York, NY USA: Kluwer Academic/Plenum Publishers, 2002, ch. 12: Mathematical modeling of lithium batteries, pp. 345-392.

[12] N. A. Chaturvedi, R. Klein, J. Christensen, J. Ahmed, and A. Kojic, "Algorithms for advanced battery-management systems," IEEE Control Systems Magazine, vol. 30, no. 3, pp. 49 - 68, 2010.

[13] J. Newman. (2008) Fortran programs for the simulation of electrochemical systems. [Online]. Available: http://www.cchem.berkeley.edu/jsngrp/fortran.html

[14] S. Zhang, K. Xu, and T. Jow, "Study of the charging process of a LiCoO2-based Li-ion battery," Journal of Power Sources, vol. 160, no. 2, pp. 1349-1354, 2006.

[15] S. S. Zhang, "The effect of the charging protocol on the cycle life of a li-ion battery," Journal of power sources, vol. 161, no. 2, pp. 1385-1391, 2006. 
[16] S. Moura, J. Stein, and H. Fathy, "Battery-Health Conscious Power Management in Plug-In Hybrid Electric Vehicles via Electrochemical Modeling and Stochastic Control," IEEE Trans. on Control Systems Technology, vol. 21, no. 3, pp. 679-694, 2013.

[17] P. Ramadass, B. Haran, P. Gomadam, R. White, and B. Popov, "Development of first principles capacity fade model for Li-ion cells," Journal of the Electrochemical Society, vol. 151, no. 2, pp. 196 - 203, 2004.

[18] A. Randall, R. Perkins, X. Zhang, and G. Plett, "Controls oriented reduced order modeling of solid-electrolyte interphase layer growth," Journal of Power Sources, vol. 209, p. 282 288, 2012.

[19] C. D. Rahn and C.-Y. Wang, Battery Systems Engineering. John Wiley \& Sons, 2012.

[20] J. C. Forman, S. Bashash, J. L. Stein, and H. K. Fathy, "Reduction of an electrochemistry-based li-ion battery model via quasi-linearization and pade approximation," Journal of the Electrochemical Society, vol. 158, no. 2, pp. A93 - A101, 2011.

[21] S. J. Moura, N. Chaturvedi, and M. Krstic, "Adaptive PDE Observer for Battery SOC/SOH Estimation via an Electrochemical Model," ASME Journal of Dynamic Systems, Measurement, and Control, vol. 136, no. 1, pp. $011015-011026$, Oct 2014.
TABLE IV

NOMENCLATURE.

\begin{tabular}{|c|c|}
\hline & Description [unit] \\
\hline$L^{ \pm}$ & Thickness of Anode/Cathode [m] \\
\hline$L^{s e p}$ & Thickness of Separator $[\mathrm{m}]$ \\
\hline$R_{s}^{ \pm}$ & Radius of Solid Particles in Anode/Cathode [m] \\
\hline$\epsilon_{e}$ & Volume Fraction in Electrolyte \\
\hline$a_{s}$ & Specific Interfacial Surface Area $\left[\mathrm{m}^{2} / \mathrm{m}^{3}\right]$ \\
\hline$D_{s}^{ \pm}$ & Diffusion Coefficient for Solid in Anode/Cathode $\left[\mathrm{m}^{2} / \mathrm{s}\right]$ \\
\hline$D_{e}$ & Diffusion Coefficient for Electrolyte $\left[\mathrm{m}^{2} / \mathrm{s}\right]$ \\
\hline$\sigma^{ \pm}$ & Conductivity of Solid in Anode/Cathode $[1 / \Omega-\mathrm{m}]$ \\
\hline$t_{c}^{0}$ & Transference Number \\
\hline$F$ & Faraday's Constant $[\mathrm{C} / \mathrm{mol}]$ \\
\hline$R$ & Gas Constant $[\mathrm{J} / \mathrm{mol}-\mathrm{K}]$ \\
\hline$\alpha_{a}$ & Charge Transfer Coefficient for Anode \\
\hline$\alpha_{c}$ & Charge Transfer Coefficient for Cathode \\
\hline$R_{f}^{ \pm}$ & Film Resistance $\left[\Omega \mathrm{m}^{2}\right]$ \\
\hline$k^{ \pm}$ & Reaction Rate in Anode/Cathode $\left[\left(\mathrm{A} / \mathrm{m}^{2}\right)\left(\mathrm{mol}^{3} / \mathrm{mol}\right)^{(1+\alpha)}\right]$ \\
\hline$c_{s, \max }^{ \pm}$ & Max Concentration in Anode/Cathode $\left[\mathrm{mol} / \mathrm{m}^{3}\right]$ \\
\hline$f_{c / a}$ & Mean Molar Activity Coefficient in Electrolyte \\
\hline$c_{p}$ & Heat Capacity $[\mathrm{J} / \mathrm{kg}-\mathrm{K}]$ \\
\hline$h_{\text {cell }}$ & Heat Transfer Coefficient $\left[\mathrm{W} / \mathrm{K}-\mathrm{m}^{2}\right]$ \\
\hline$T_{a m b}$ & Ambient Temperature $[\mathrm{K}]$ \\
\hline$\rho^{a v g}$ & Lumped Cell Density $\left[\mathrm{kg} / \mathrm{m}^{3}\right]$ \\
\hline$\theta_{\min }^{ \pm}$ & Minimum Normalized Concentration in Anode/Cathode \\
\hline$\theta_{\max }^{ \pm}$ & Maximum Normalized Concentration in Anode/Cathode \\
\hline$c_{e_{m i n}}$ & Minimum Electrolyte Concentration $\left[\mathrm{mol} / \mathrm{m}^{3}\right]$ \\
\hline$c_{e_{\max }}$ & Maximum Electrolyte Concentration $\left[\mathrm{mol} / \mathrm{m}^{3}\right]$ \\
\hline$T_{\min }$ & Minimum Bulk Cell Temperature $[\mathrm{K}]$ \\
\hline$T_{\max }$ & Maximum Bulk Cell Temperature $[\mathrm{K}]$ \\
\hline$U_{s}$ & Side Reaction Equilibrium Potential [V] \\
\hline$c_{s}^{ \pm}$ & Lithium Concentration in the Solid $\left[\mathrm{mol} / \mathrm{m}^{3}\right]$ \\
\hline$c_{e}$ & Lithium Concentration in the Electrolyte $\left[\mathrm{mol} / \mathrm{m}^{3}\right]$ \\
\hline$c_{s s}^{ \pm}$ & Concentration at Particle Surf. in Anode/Cathode $\left[\mathrm{mol} / \mathrm{m}^{3}\right]$ \\
\hline$U^{ \pm}$ & Equilibrium Potential in Anode/Cathode [V] \\
\hline$\eta^{ \pm}$ & Overpotential $[\mathrm{V}]$ \\
\hline$\eta_{s}$ & Side Reaction Overpotential [V] \\
\hline$\phi_{s}^{ \pm}$ & Solid Electric Potential [V] \\
\hline$\phi_{e}$ & Electrolyte Electric Potential [V] \\
\hline$i_{e}^{ \pm}$ & Ionic Current $\left[\mathrm{A} / \mathrm{m}^{2}\right]$ \\
\hline$j_{n}^{ \pm}$ & Molar Ion Fluxes $\left[\mathrm{mol} / \mathrm{m}^{2}-\mathrm{s}\right]$ \\
\hline$T$ & Bulk Cell Temperature $[\mathrm{K}]$ \\
\hline$I$ & Applied Current $\left[\mathrm{A} / \mathrm{m}^{2}\right]$ \\
\hline$I^{r}$ & Reference Current $\left[\mathrm{A} / \mathrm{m}^{2}\right]$ \\
\hline$\beta$ & MRG Reference Current Scaling Factor \\
\hline$i_{0}^{ \pm}$ & Exchange Current Density $\left[\mathrm{A} / \mathrm{m}^{2}\right]$ \\
\hline $\bar{c}_{s}^{ \pm}$ & Particle Vol. Avg. Concentration in Anode/Cathode $\left[\mathrm{mol} / \mathrm{m}^{3}\right]$ \\
\hline$\theta^{ \pm}$ & Normalized Concentration in Anode/Cathode \\
\hline
\end{tabular}

